\title{
Alpha Thalassemia Trait
}

National Cancer Institute

\section{Source}

National Cancer Institute. Alpha Thalassemia Trait. NCI Thesaurus. Code C95535.

A condition in which a person has reduced protein production from two of the four alphaglobin alleles. 